\title{
Characterization of Si Nanostructured Surfaces
}

\author{
Saleem H. Zaidi ${ }^{a}$, James M. Gee ${ }^{b}$, Douglas S. Ruby ${ }^{b}$, and S. R. J. Brueck ${ }^{a}$ \\ ${ }^{a}$ Center For High Technology Materials, University of New Mexico \\ 1313 Goddard, SE Albuquerque, NM 87106
}

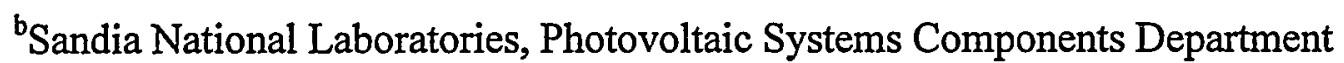

P. O. Box 5800, MS 0752, Albuquerque, NM 87185-0752

\begin{abstract}
Surface texturing of Si to enhance absorption particularly in the IR spectral region has been extensively investigated. Previous research chiefly examined approaches based on geometrical optics. These surface textures typically consist of pyramids with dimensions much larger than optical wavelengths. We have investigated a physical optics approach that relies on surface texture features comparable to, or smaller than, the optical wavelengths inside the semiconductor material. Light interaction at this are strongly dependent on incident polarization and surface profile. Nanoscale textures can be tuned for either narrow band, or broad band absorptive behavior. Lowest broad band reflection has been observed for triangular profiles with linewidths significantly less than $100 \mathrm{~nm}$. Si nanostructures have been integrated into large $\left(\sim 42 \mathrm{~cm}^{2}\right)$ area solar cells. Internal quantum efficiency measurements in comparison with polished and conventionally textured cells show lower efficiency in the UV-visible (350-680 nm), but significantly higher IR (700-1200 nm) efficiency.
\end{abstract}

Keywords: Si Nanostructures, Solar cells, Reactive Ion Etching of Si

\section{INTRODUCTION}

Surface texturing aimed at enhanced absorption in Si has been extensively investigated. Si has a large absorption coefficient in the visible, albeit, it also has a high reflection coefficient ${ }^{1}$. Reflection losses are reduced by applying anti-reflection (AR) films to a Si surface ${ }^{2}$. AR films have a resonant structure that limits their effectiveness to a narrow range of angles and wavelengths. An effective alternative has been geometrical texturing ${ }^{3}$ of a Si surface combined with appropriate AR film stack. Besides reducing the external reflectance, geometrical texturing schemes also improve absorption near the bandgap by obliquely coupling light into the semiconductor. Si exhibits weak absorption near the band edge because it has an indirect transition bandgap. The oblique coupling of light improves optical absorption by increasing the optical path length. Geometrical texturing schemes can also improve optical absorption in thin Si samples by means of "light trapping." Light trapping refers to enhanced optical absorption through the confinement of light within the semiconductor. Optical confinement occurs when the obliquely coupled light is incident upon a surface at an angle greater than the critical angle. Using geometrical optics considerations, the maximum enhancement in optical absorption of a textured sheet with refractive index $\mathrm{n}$ compared to a planar sheet is $4 \mathrm{n}^{2} 4$.

Enhanced optical absorption due to textured surfaces can also be analyzed with statistical optics. Using this approach, Yablonovitch found the same result that the effective absorption can be enhanced by as much as a factor of $4 n^{2}$ over a planar sheet ${ }^{5}$, where $\mathrm{n}$ is the refractive index. However, in order to reach this limit, the surface texture must fully randomize incident light to fill internal optical phase space. Deckman et al., ${ }^{6}$ have demonstrated that optimum random textures have dimensions slightly larger than the wavelength of light in the material. If the lateral dimensions of the microstructure are too large, light is specularly reflected reducing internal randomization, and if the lateral dimensions are much smaller than the wavelength of light, scattering is not effective again reducing internal randomization. Therefore, in order to optimize absorption, a precise balance between randomness and microstructure dimensions has to be maintained. If instead of a random surface, a periodically textured surface such as a grating is used, the absorption may be enhanced beyond this statistical limit over a limited spectral range. The basic physics of this approach has been described by Sheng et al., ${ }^{7}$ in terms of a rough analogy between interactions of photons with a periodic structure, and interactions of electrons with a periodic lattice in a crystal. The effect of the periodic structure is to create peaks in the particle density of states at one set of frequencies at the expense of gaps at the others keeping total integrated number of states fixed. By choosing grating parameters such that the gap in $\rho(\omega)$ falls below the absorption edge, peaks in $\rho(\omega)$ above it, photon states from non-absorbing region are transferred to the absorbing region. In this way, $\rho(\omega)$ can be enhanced beyond the statistical limit. 


\section{DISCLAIMER}

This report was prepared as an account of work sponsored by an agency of the United States Government. Neither the United States Government nor any agency thereof, nor any of their employees, make any warranty, express or implied, or assumes any legal liability or responsibility for the accuracy, completeness, or usefulness of any information, apparatus, product, or process disclosed, or represents that its use would not infringe privately owned rights. Reference herein to any specific commercial product, process, or service by trade name, trademark, manufacturer, or otherwise does not necessarily constitute or imply its endorsement, recommendation, or favoring by the United States Government or any agency thereof. The views and opinions of authors expressed herein do not necessarily state or reflect those of the United States Government or any agency thereof. 


\section{DISCLAIMER}

Portions of this document may be illegible in electronic image products. Images are produced from the best available original document. 
We have investigated light interaction with nanoscale periodic and random structures etched in Si using conventional reactive ion etching techniques ${ }^{8}$. These structures are of interest due to their enhanced absorption, low diffraction losses, and the ability to tailor the spectral response from UV to IR region. In section 2, we describe techniques to form uniform periodic structures in Si using lithography and the wet and dry etching techniques along with evaluation of their spectral reflectance response. In section 3, we present techniques for maskless random reactive ion etching in Si to form nanoscale features exhibiting broadband absorptive behavior. In section 4 , some device results are presented, and section 5 summarizes the results.

\section{INTERFEROMETRIC LITHOGRAPHY}

We have investigated interferometric lithography (IL) as a cost-effective alternative in printing nanoscale features. IL techniques have been demonstrated to produce uniform nm-scale structures over large areas at low cost. The feature dimension for $\mathrm{IL}$ is $1 / 2$ of the interference period $\lambda / 2 \sin \theta$, where $2 \theta$ is the intersection angle between the two exposing laser beams. For readily available laser sources from $0.488-0.193 \mu \mathrm{m}$ at $\theta=75^{\circ}$, this translates into periods $\sim 0.25-0.1 \mu \mathrm{m}$ with linewidths of $\sim 0.12-0.05 \mu \mathrm{m}$. Single exposure interferometric grating fabrication has a long history 9 . Figure 1 shows a typical experimental configuration in which an expanded and collimated laser beam is incident on a Fresnel mirror (FM) arrangement mounted on a rotation stage for period variation ${ }^{10}$. The mirror and sample stages have tilt \& tip adjustments, the sample stage is equipped with in-plane rotation adjustment as well. The interference between two beams, the incident, and the reflected, results in a periodic pattern, whose pitch can be precisely varied by the computer-controlled rotation stage. Grating patterns are first formed in photoresist followed by a pattern transfer to the underlying substrate. Figure 2 shows two examples of $360-\mathrm{nm}$ and $250-\mathrm{nm}$ period photoresist gratings fabricated on an anti-reflection film on a Si wafer. These structures were fabricated with laser exposure at $\lambda=355 \mathrm{~nm}$ in Shipley $505-A$ positive photoresist. The grating linewidths were $\sim 180$ and $130 \mathrm{~nm}$ respectively at a height $\sim 500 \mathrm{~nm}^{11}$.

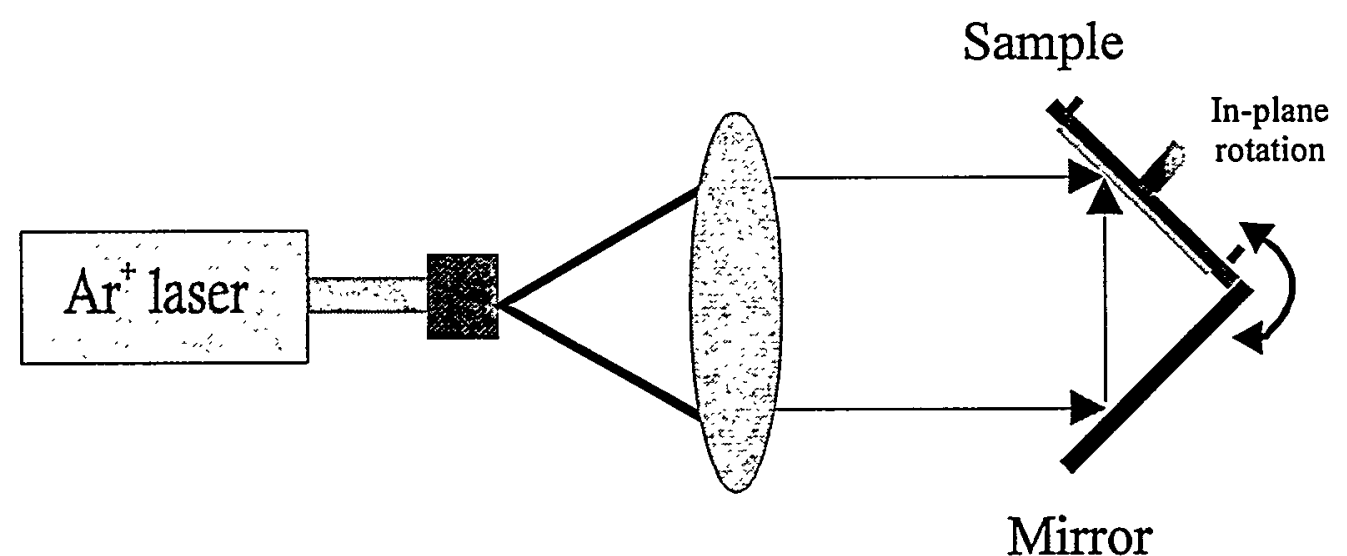

Figure 1 Typical experimental configuration for interferometric lithography.
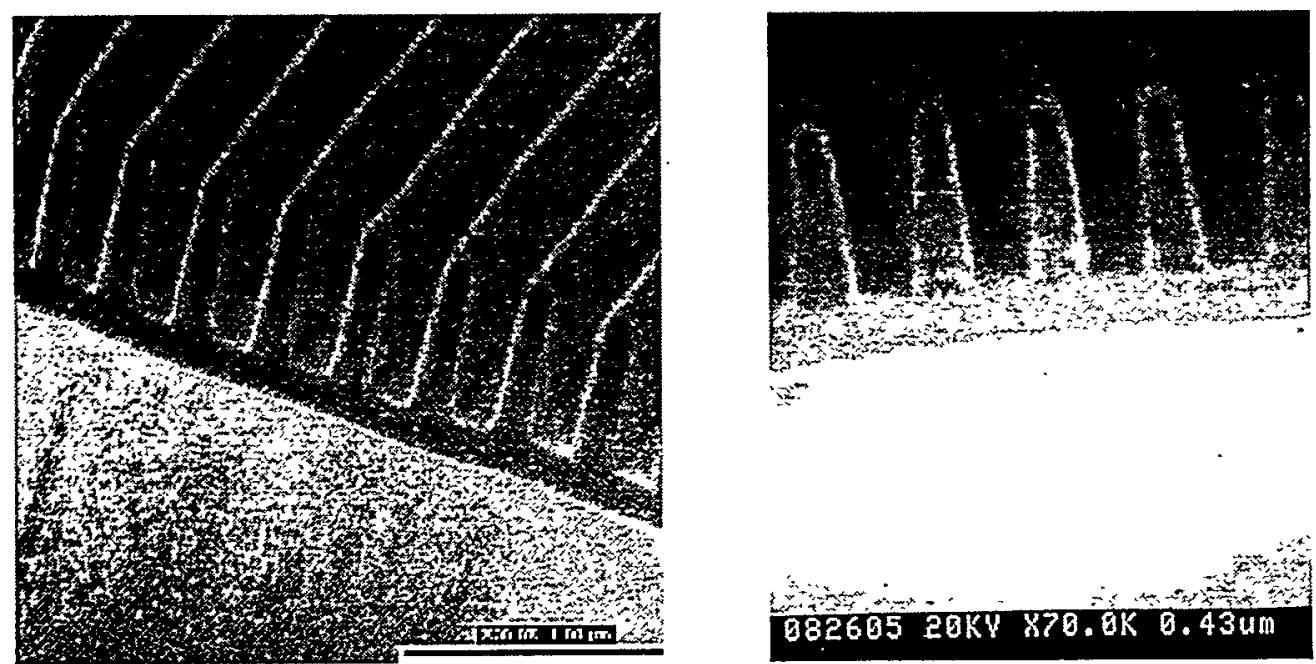

Figure 2 Cross-sectional photoresist profiles of a $360-\mathrm{nm}$ (left), and $250-\mathrm{nm}$ (right) pitch gratings. 

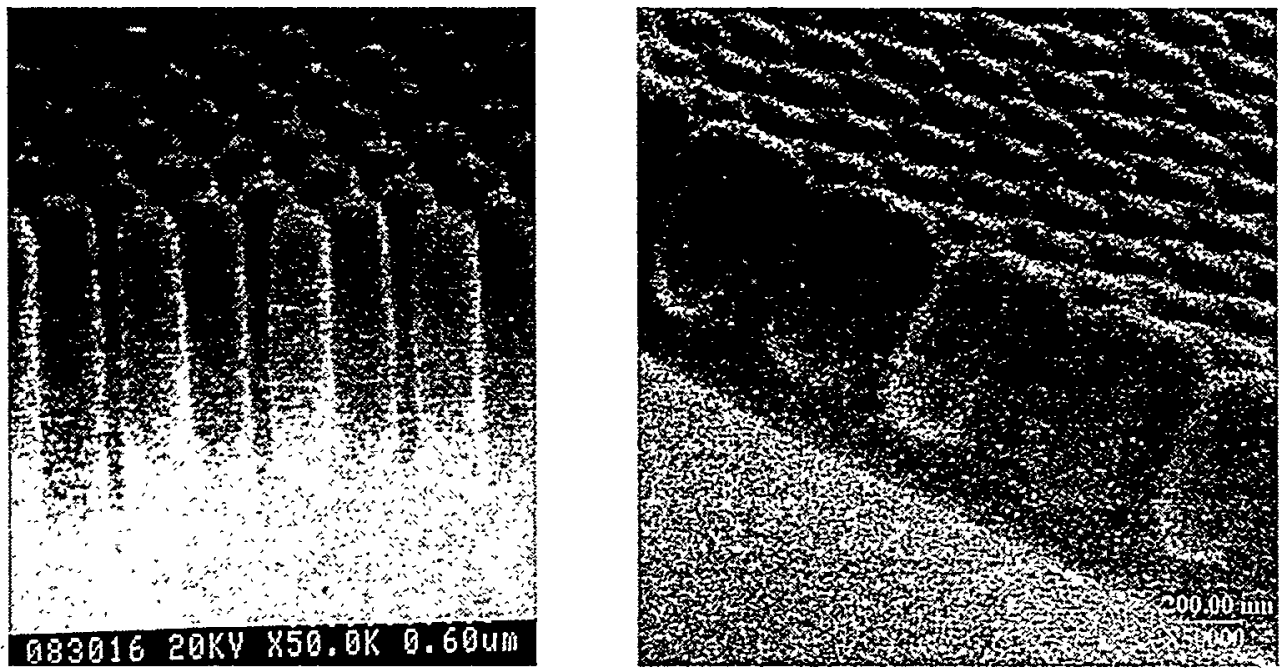

Figure 3 Cross-sectional profiles of a 500-nm (left), and 360-nm (right) pitch posts and vias formed in positive and negative photoresist respectively.

Single exposure IL is limited in its applicability to 1-D structures, more useful structures can be formed using multiple exposures either with a simple 2-beam configuration, or using multiple laser beams ${ }^{12}$. Figure 3 shows simplest examples of 2D patterns consisting of posts and vias formed in Shipley 508 positive and Ultra i-300 negative photoresists respectively by two IL exposures at $90^{\circ}$ in the same level of the resist. Multiple exposure interferometric lithography (MEIL) techniques appropriately combined with a synthesis procedure offer a cost-effective alternative to conventional optical lithography in nm-scale regime. A major limitation of these methods remains overall periodic character of the patterns; this can be addressed by mix-and-match with optical lithography ${ }^{13}$ or by imaging interferometric lithography ${ }^{14}$. In either case, development of an understanding and process for matching multiple exposures is a critical step in the development of these capabilities.

\section{1-D and 2-D Si Nanostructures and Their Spectral Reflectance Response}

The photoresist structures shown in Figs. 2-3 are used to transfer pattern to etch mask film for Si etching. We have investigated both wet ${ }^{15}$ and reactive etching techniques (RIE) in $\mathrm{Si}^{16}$. The $\mathrm{RIE}$ experiments were carried out at room temperature in a Techniques PEII-A parallel plate reactor using $\mathrm{SF}_{6} / \mathrm{O}_{2}$ base plasma chemistry. Optimum etching parameters were obtained at power of $\sim 50 \mathrm{~W}, \mathrm{SF}_{6} / \mathrm{O}_{2}$ flow ratio of $\sim 1$, and pressure of $\sim 180 \mathrm{mTorr}$. For most of the work reported here, a thin $(\sim 30 \mathrm{~nm}) \mathrm{Cr}$ film was used as an etch mask during RIE. Figure 4 shows a scanning electron microscope (SEM) picture of 870-nm period grating etched in Si to a depth of $\sim 1000 \mathrm{~nm}$ at a linewidth $\sim 250 \mathrm{~nm}$. Also shown in Fig. 4 are the normal-incidence spectral reflectance measurements from this 1-D grating structure. The terms TE \& TM refer to electric field parallel, and perpendicular to the grating lines. For comparison, the grating reflectance has been compared with polished Si surface reflection under identical conditions. The Si reflectance has been normalized to unity, and the same normalization factor has been applied to the grating reflectance data. These measurements demonstrate the spectral reflection is polarization
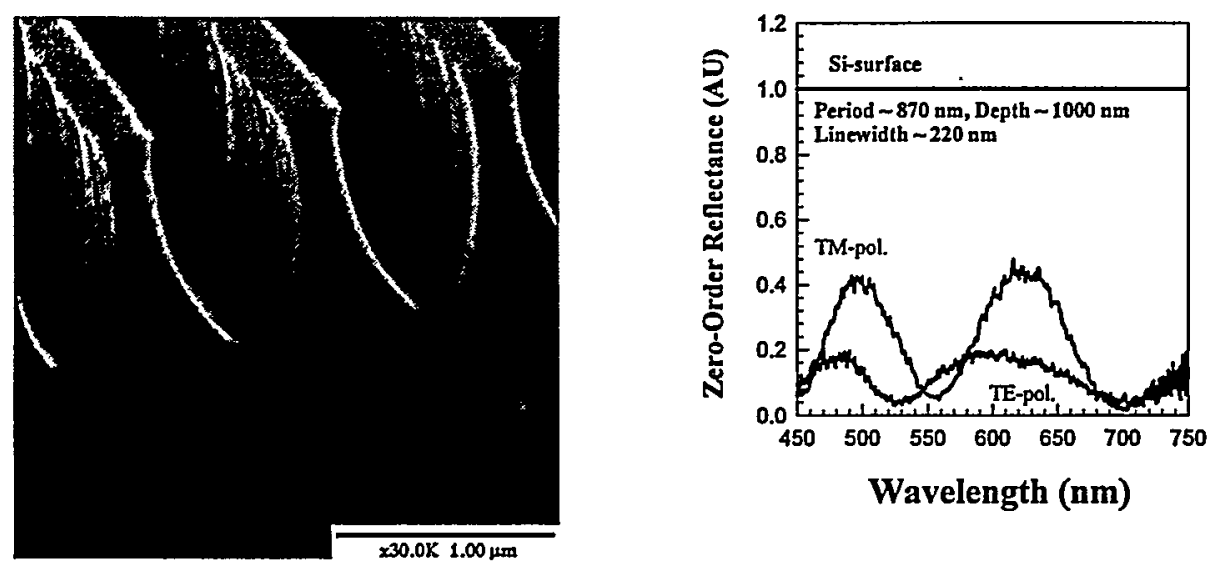

Figure 4 SEM picture of 870-nm period grating in Si (left), and its spectral reflectance response (right). 
dependent. For the TE-polarization, there are two reflection minima at $\sim 4 \%$ of the Si baseline at $\sim 525 \& 700 \mathrm{~nm}$. For TMpolarization, the reflection minima at $\sim 6-4 \%$ of the Si baseline are at $\sim 450,560 \& 700 \mathrm{~nm}$ respectively. At $\sim 550-\mathrm{nm}$, the bulk Si reflectivity is $\sim 0.364$, therefore, a $4 \%$ grating reflection corresponds to a reflectivity of $\sim 0.0146$. It should be pointed out that there are diffraction orders that carry some of the energy at this grating period, but that very little energy is carried by these diffraction orders due to grating depth $\sim 1000 \mathrm{~nm}$. Use of the higher diffraction orders may be advantageous for enhancing the optical absorption since these are transmitted at increasingly large angles. The wavelength-selective absorption of the gratings can be tailored for various spectral ranges by modifying their parameters. Figure 5shows the SEM picture of a 500-nm period gating and its spectral reflectance response. Notice that at wavelengths $>500 \mathrm{~nm}$, there are no diffraction losses. The grating structure shown in Fig. 5 was formed by preferential $\mathrm{KOH}$ etching of (110) Si ${ }^{14}$. The reflectance measurements in Fig. 5 show low narrow absorption bands at $\sim 680$ and $780 \mathrm{~nm}$. The reflection is low for both polarizations in 400-550 nm regions. We have also investigated spectral reflectance of 2-D structures. These structures are interest due to their polarization-independent response. Figure 6 shows SEM picture of a 2-D post pattern etched to a depth of $\sim 800 \mathrm{~nm}$ at a linewidth of $\sim 300 \mathrm{~nm}$, also shown is its spectral reflectance response. This structure shows broadband reduced reflection in comparison with polished $\mathrm{Si}$, weak minima in reflection are seen at $\sim 450,550$, and $750 \mathrm{~nm}$. The reflectance measurements in Figs. 4-6 demonstrate the narrow band absorptive behavior of rectangular-profiled grating structures. We have also investigated the spectral reflection response of triangular-profiled grating structures. Figure 7 shows SEM picture of a 400-nm period grating and its spectral reflectance response. This structure has almost zero, polarization-independent reflection. Due to sloped sidewalls, the linewidths vary from $\sim 10-20 \mathrm{~nm}$ at the top to $\sim 130 \mathrm{~nm}$ at the midpoint, overall depth is $\sim 700 \mathrm{~nm}$. The reduced reflection from such a structure may be attributed to a gradual variation in effective refractive index from the top (air 1) to the bottom $(\mathrm{Si})^{17}$.

We have investigated modeling of the experimental data using commercially available grating software GSOLVER ${ }^{\mathrm{TM}}$. The calculated spectral reflectance has been difficult to match with the experimental data perhaps due to strong dependence on grating parameters such as linewidth, depth, and slope of the grating line. The model calculations are in good qualitative agreement with the basic features of the wavelength-selective behavior of rectangular-profiled gratings, and broad-band absorptive behavior of triangular-profiled grating structures. Modeling efforts are underway for a precise match with arbitrary-shaped 1-D and 2-D grating structures.
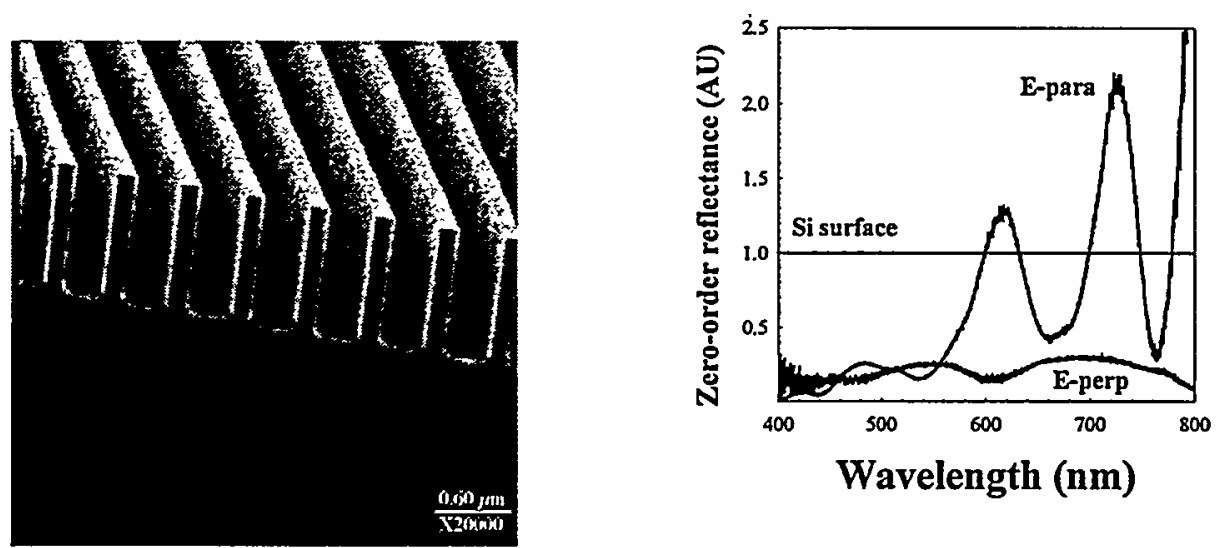

Figure 5 SEM picture of a 130-nm linewidth, 1000-nm deep grating (right), and its spectral reflection response (left).
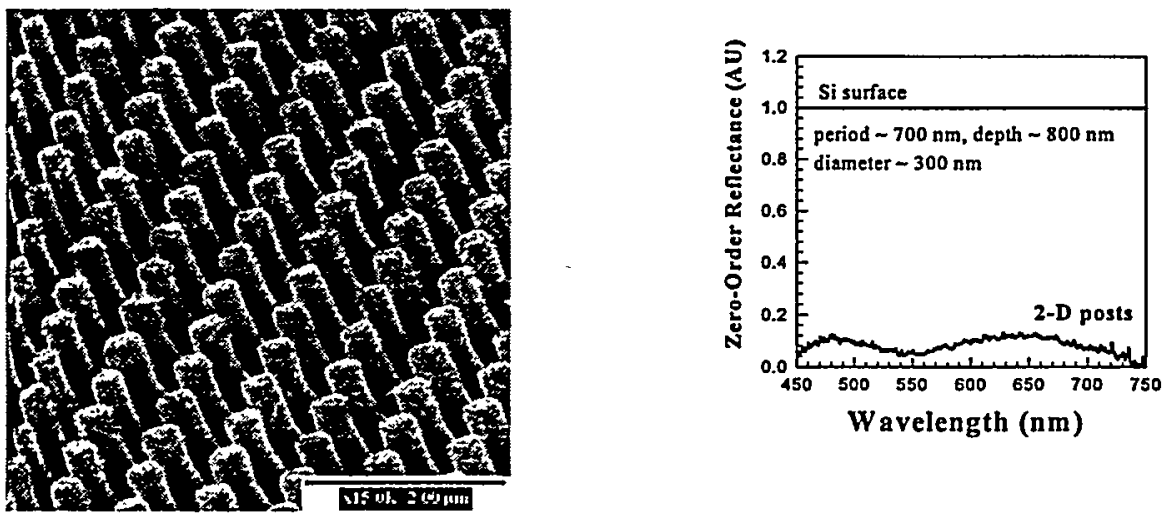

Figure 6 SEM picture of a 2-D post pattern (left), and its spectral reflectance response (right). 

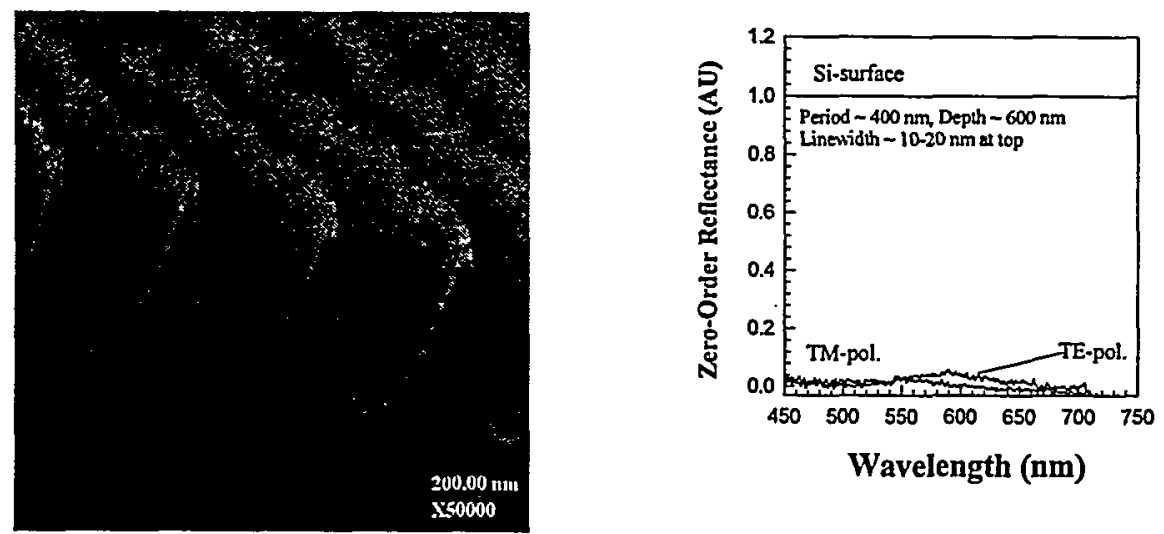

Figure 7 SEM picture of a 1-D triangular-profiled grating (left), and its spectral reflectance response (right).

\section{RANDOMLY TEXTURED NANOSCALE SURFACES}

The interferometric lithography based texturing techniques offer flexibility in tuning reflectance response. For most terrestrial solar cell applications, broadband reduced reflection is required. The RIE techniques described above have been applied to form randomly etched absorptive structures. The random nanoscale texturing for reduced reflection was first reported by Gittleman et al., ${ }^{18}$ in a maskless RIE process. Si reflection was measured to be less than $0.001 \%$ in the visible region. These structures had lateral dimensions of $\sim 100-300 \mathrm{~nm}$, and depths $\sim 0-2000 \mathrm{~nm}$. Similar structures formed by reactive ion etching were also reported by Craighead et al., ${ }^{19}$ in thin Si films. More recently, Jansen et al., ${ }^{20}$ have reported on RIE schemes to form a black Si șurface consisting of high aspect ratio nanoscale columnar structures. It is generally recognized that reactive ion etching of $\mathrm{Si}$ can yield almost black surfaces, however, no systematic research effort has been directed at large area fabrication of these surfaces.

We have investigated random texturing of Si aimed at reduced Si reflection in the visible to IR spectral regions. In our initial experiments, small $(\sim 1-2 \mathrm{~cm})$ Si samples were mounted on an $\mathrm{Al}$ substrate inside the RIE chamber. Figure 8 shows normal incidence spectral reflectance of Si surface as a function of the etching time, for comparison, reflection from a polished $\mathrm{Si}$ surface under identical conditions had been normalized to unity, and same normalization factor was applied to reflection data from the etched surfaces. From Fig. 8, it is seen that substantial reflection reduction is achieved after 15-minutes of etching (at least $\sim 1.5-\mu \mathrm{m}$ thickness of Si removed). The etched surfaces show almost zero reflection in the visible and only slightly higher reflection in the IR region. At $\lambda \sim 600-\mathrm{nm}$, the reflection from the 30-min etched sample is reduced by a factor of 85 relative to bare $\mathrm{Si}$. Assuming $\sim 0.33$ absolute $\mathrm{Si}$ reflectance, this represents absolute Si reflection of $\sim 0.004$ without applying

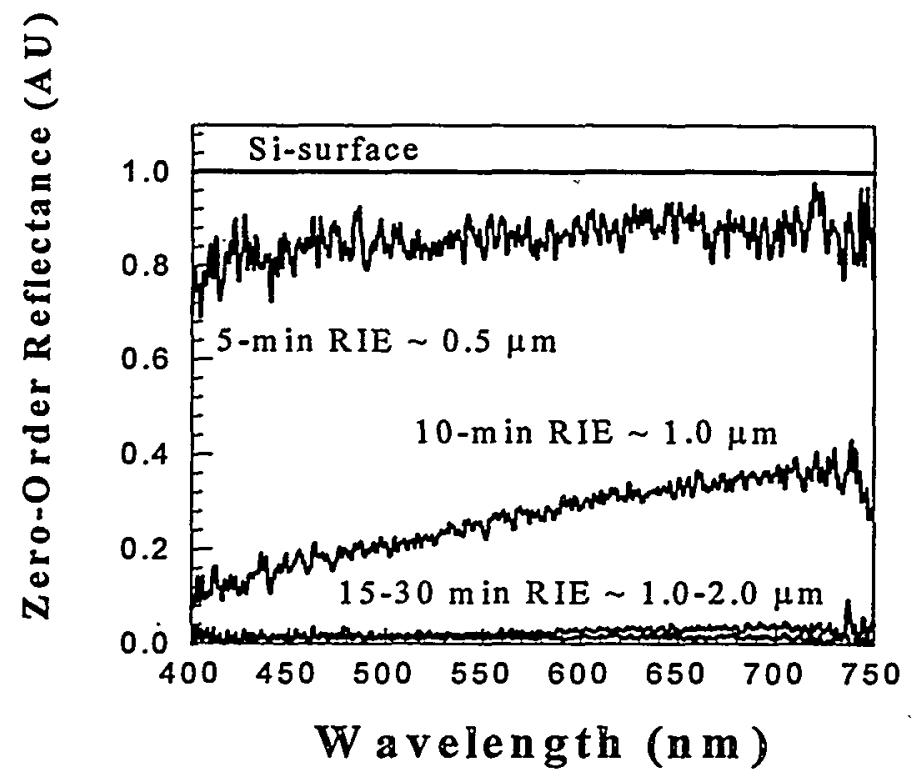

Figúre 8 Normal incidence spectral reflectance from randomly etched Si structures. 
anti-reflection films. The 15-30 min RIE samples appear almost black at normal incidence, and at large incident angles show slightly higher bluish reflectance. We have carried out scanning electron microscope (SEM) investigation of these randomly etched surfaces. It is seen that the absorptive texture consists of densely packed, random inverted pyramids, with lateral dimensions of $\sim 100-500 \mathrm{~nm}$, and depths $\sim 0-1000 \mathrm{~nm}$.

For photovoltaic device applications large area application of these random texturing schemes is desirable. We investigated texturing of 4 " wafers under identical conditions. The reactive ion etching processes failed to form absorptive surface structure. From visual inspection of 4" wafers, edges appeared to have strong dark texture, whereas the center did not exhibit any texture. This suggested that during RIE process, microscopic Al particles are sputtered and deposited on Si to act as random etch masks. These Al particles don't have enough mobility to travel to the wafer center so that no appreciable texture is developed. In order to verify this, we etched two Si samples at the same time. One sample was mounted on $\mathrm{Al}$, other on the RIE chamber surface made of Copper-coated surface. After 30 minutes of etching, both samples were removed from the chamber. The sample on Al holder had developed a black texture, whereas the sample without Al base did not exhibit a significant texture. Figure 9 shows normal incidence spectral reflectance measurements on these two samples, for comparison Si reflectance under identical conditions normalized to unity is also shown. It is seen that sample on Al substrate shows broadband reduced reflection similar to that observed in Fig. 8, whereas the Si sample without Al base shows only $40 \%$ reduction in reflection in comparison with polished Si. Figures 10 \& 11 show SEM pictures of texture on absorptive and non-absorptive samples. It is seen that non-absorptive texture consists of random distribution of 10-50 nm lateral features without any significant depth. The absorptive structure has a random distribution of pyramidal structures with typical dimensions $\sim 100-1000 \mathrm{~nm}$, and depths $\sim 1000 \mathrm{~nm}$. Comparison of feature dimensions in Figs. $10 \& 11$ shows that reduced reflection is accomplished by high aspect ratio nanoscale $(\sim 100-500 \mathrm{~nm})$ feature sizes with triangular slopes. These structures are similar to the 1-D grating profile shown in Fig. 7.

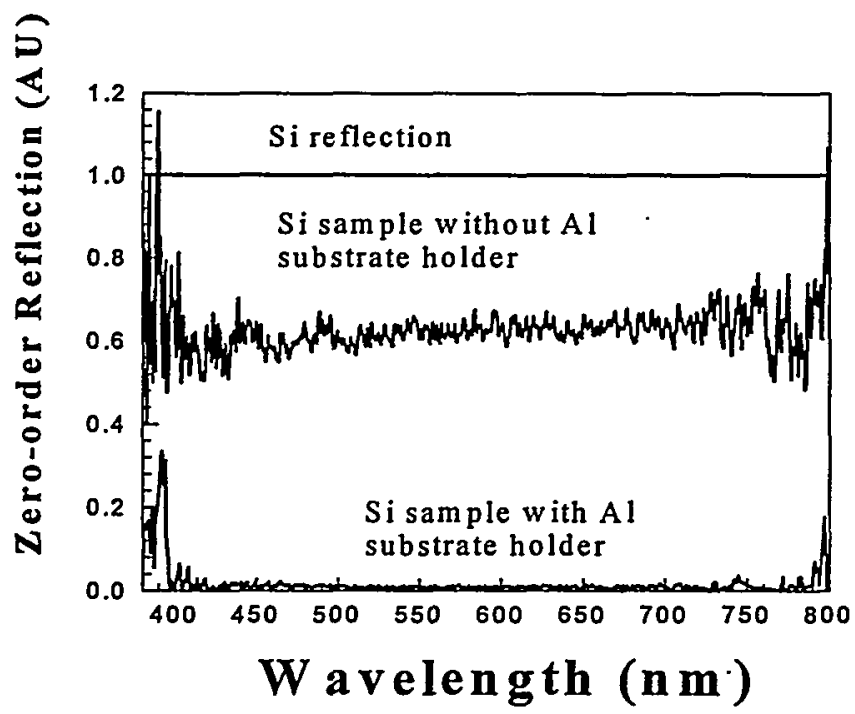

Figure 9 Normal incidence spectral reflectance from randomly etched Si structures without and without Al holders.

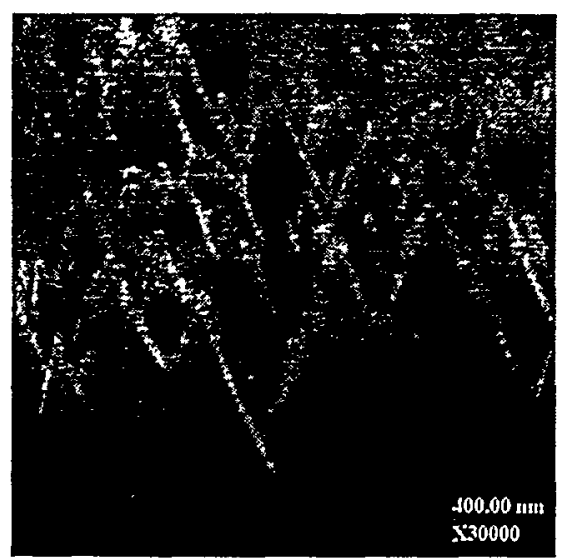

Figure 10 SEM picture of a black Si RIE-textured surface.

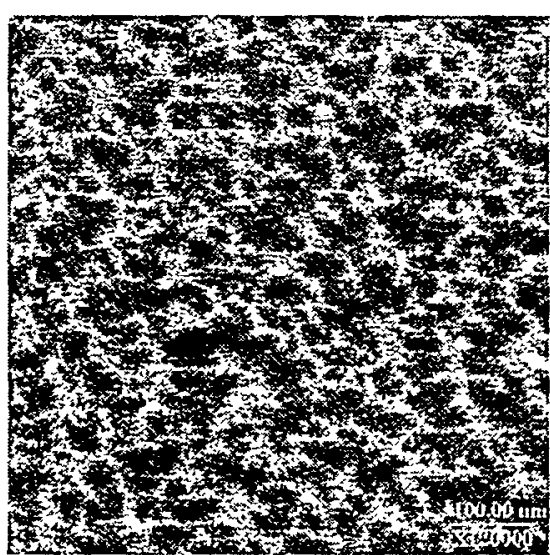

Figure 11 SEM picture of a lightly absorptive Si RIEtextured surface. 
We have demonstrated that $\mathrm{Si}$ absorptive texture in an RIE chamber is a result of micro-masking by sputtered Al particles. Small Al particles can be identified at the top of the pyramids in Fig. 10. For large area texturing, an alternate approach is required that can deposit a random etch mask on a single, or multi-crystalline surface. One such approach is natural lithography developed by Deckman et al., ${ }^{21}$. In this approach, sub- $\mu \mathrm{m}(\sim 0.1-0.8 \mu \mathrm{m})$ silica spherical particles are spin-coated onto the semiconductor surface, and act as etch RIE masks. We have been able to develop an alternate texturing scheme that is effective in forming black Si surfaces. A thin $(\sim 5 \mathrm{~nm})$ island $\mathrm{Cr}$ film is deposited by electron beam sputtering, and acts as an etch mask to generate absorptive nanoscale features. Further work is in progress aimed at optimizing this method.

\section{NANOSCALE TEXTURED SOLAR CELLS}

We have applied nanoscale-texturing schemes on 4 " polished Si wafers. These p-type wafers were first textured, and then diffused with phosphorous. Solar cells were fabricated on an area of $42 \mathrm{~cm}^{2}$. For comparison with RIE-textured cells, polished, and conventionally wet-chemically textured cells were also fabricated under identical conditions. Figure 12 shows IQE measurements on randomly the RIE textured, the control and the wet-chemically polished Si solar cells fabricated and tested at Sandia National Laboratories. Randomly textured Cell was formed by depositing a thin $(\sim 5 \mathrm{~nm}) \mathrm{Cr}$ film on Si. This island $\mathrm{Cr}$ film was then used as an etch mask for nanoscale texturing of top Si surface. The salient features of these IQE measurements are listed below:

a) Highest efficiency has been obtained for the randomly textured solar cell, b) Randomly textured cell has lower IQE in 300-700-nm spectral region than either the control, or chemically-textured cell,
and c) Randomly textured cell has higher IQE in 700-1200-nm spectral region than either the control, or chemically textured
cell.

It is known that reactive ion etching techniques produce surface damage 22 . Various surface passivation techniques have been investigated in reducing RIE damage ${ }^{23}$. For the solar cell devices shown in Fig. 12, surface passivation treatment consisted of a thin $(\sim 10 \mathrm{~nm})$ thermally grown oxide film ${ }^{24}$. The UV response of RIE cell fabricated using is lower than either the chemically textured, or the control cell, we expect to improve IQE of RIE-textured cell by additional passivation treatments. We are also in the process of integrating periodically textured surfaces into solar cells.

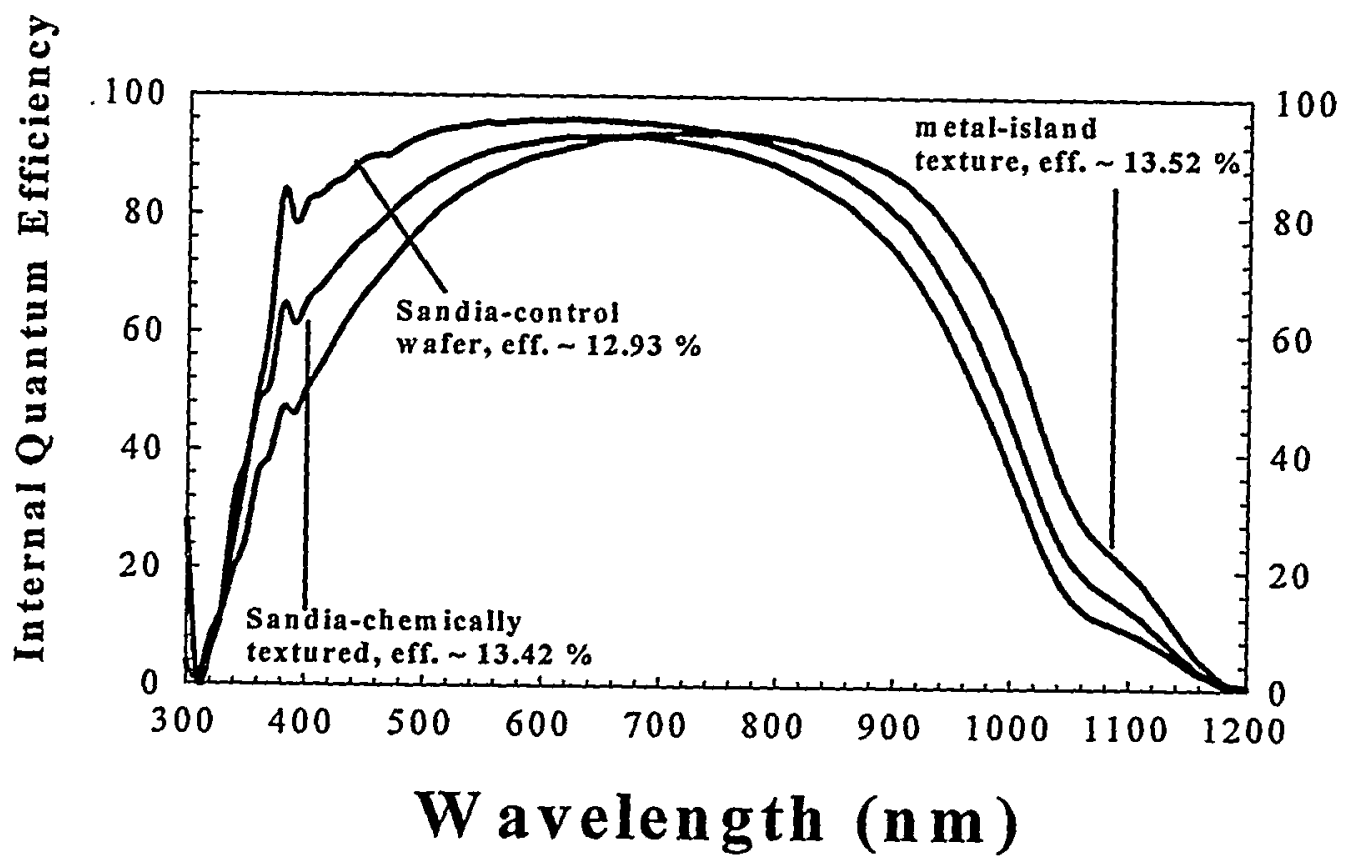

Figure 12 Internal Quantum efficiency measurements on randomly textured solar cells. 


\section{SUMMARY}

We have demonstrated large area, low-cost patterning of Si nanostructures. Interferometric lithography offers a unique a unique manufacturing capability of a wide range of 1-D and 2-D grating structures. Reactive ion etching techniques offer flexibility in etching a variety of profiles. Rectangular and triangular profiles are respectively characterized by narrowband and broadband absorption behavior. Random RIE-generated nanostructures exhibit broadband absorptive over wide angular and spectral variations. Integration of these structures into large area solar cells has demonstrated a significant improvement in internal quantum efficiency in 700-1100 nm spectral range.

\section{ACKNOWLEDGEMENTS}

Partial support for this work from Sandia National Laboratories under contract \# BB-7211 is appreciated.

\section{REFERENCES}

1. D. E. Aspness and A. A. Studna, "Dielectric functions and optical parameters of $\mathrm{Si}, \mathrm{Ge}, \mathrm{GaP}, \mathrm{GaAs}, \mathrm{GaSb}, \mathrm{InP}$, InAs, and InSb from 1.5 to $6.0 \mathrm{eV}$," Phys. Rev. B 27, 985, 1983.

2. B. O. Seraphin and A. B. Meinel, Optical Properties of Solids, North-Holland, Amesterdam, 1974.

3. P. Campbell and M. A. Green, "Light trapping properties of pyramidally textured surfaces," J. Appl. Phys. 62, 243, 1987.

4. James M. Gee, "The effect of parasitic absorption losses on light trapping in thin silicon solar cell," $12^{\text {th }}$ PVSC, pp. 549$554,1988$.

5. E. Yablonovitch," Statistical ray optics," 899, 1982.

6. H. W. Deckman, C. R. Wronski, H. Witzke, and E. Yablonovitch, "Optically enhanced amorphous silicon solar cells," Appl. Phys. Lett. 42, 968, 1983.

7. Ping Sheng, A. N. Bloch, and R. S. Stepleman, "Wavelength-selective absorption enhancement in thin-film solar cells," Appl. Phys. Lett. 43, 579, 1983.

8. Y. Tzeng and T. H. Lin, "Dry etching of Si materials in $\mathrm{SF}_{6}$ based plasmas, " J. Electrochem. Soc. 134, 2304, 1987.

9. L. F. Johnson, G. W. Kammlott, and K. A. Ingersoll, "Generation of periodic surface corrugations," Appl. Opt. 17, pp. 1165-1181, 1978.

10. Saleem H. Zaidi and S. R. J. Brueck, "High aspect ratio holographic photoresist gratings," Appl. Opt. 27, 2999, 1988.

11. Saleem H. Zaidi, S. R. J. Brueck, F. M. Schellenberg, R. S. Mackay, K. Uekert, and J. J. Persoff, "Interferometric lithography exposure tool for 180-nm structures," SPIE 3048, pp. 248-254, 1997.

12. Saleem H. Zaidi and S. R. J. Brueck, "Multiple exposure interferometric lithography," Jour. Vac. Sci. Technol. B 11, $653,1993$.

13. Saleem H. Zaidi, S. R. J. Brueck, T. Hill, and R. N. Shagam, "Mix-and-match interferometric and optical lithographies for nanoscale structures," SPIE-3331, 406, 1998.

14. X. Chen and S. R. J. Brueck, "Imaging interferometric lithography: a wavelength division multiplex approach to extending optical lithography," J. Vac. Sci. Technol. B 16, pp. 3392-3397, 1998.

15. Saleem H. Zaidi, An-Shyang Chu, and S. R. J. Brueck, "Optical properties of nanoscale, one-dimensional silicon grating structures," J. Appl. Phys. 80, 6997, 1996.

16. Saleem H. Zaidi and S. R. J. Brueck, "Si-texturing with sub-wavelength structures," $26^{\text {th }}$ PVSC, pp. 171-174, 1997.

17. D. H. Raguin and G. M. Morris, "Anti-reflection structured surfaces for the infra red spectral region," Appl. Opt. 32, pp. 1154-1167, 1993.

18. J. I. Gittleman, E. K. Sichel, H. W. Lehman, and R. Widmer, "Textured Si: a selective absorber for solar thermal conversion," Appl. Phys. Lett. 35, 742, 1979.

19. H. G. Craighead, R. E. Howard, and D. M. Tennant, " Textured thin-film Si solar selective absorber using reactive ion etching," Appl. Phys. Lett. 37, 653, 1980.

20. H. Jansen, M. de Boer, B. Otter, and M. Elwenroek, " The black Si method IV: the fabrication of three-dimensional structures in silicon with high aspect ratios for scanning probe microscopy and other applications," Proc. IEEE MEMS95, 88, 1995.

21. H. W. Deckman and J. H. Dunsmuir," Applications of surface textures produced with natural lithography," J. Vac. Sci. Technol. B 1, pp. 1109-1112, 1983.

22. K. Kwon, H. Park, K. Kim, C. Kim, and Y. Sung, "Recovery of silicon surface after reactive ion etching of SiO2 using CHF3/C2F6 plasma," Jpn. J. Appl: Phys. 25, Pt. 1, pp. 1611-1616, (1996).

23. H. W. Pang, D. D. Rathman. D. J. Silversmith, R. W. Mountain, and P. D. Degaff, " Damage induced in Si by ionmilling or reactive ion etching," J. Appl. Phys. 54, pp. 3272-3277, 1983.

Sandia is a multiprogram laboratory operated by Sandia Corporation. a Lockheed Martin Company. for the United States Department of Energy under contract DE-AC04.94AL85000. 

24. P. E. Gruenbaum, J. Y. Gan, R. R. King, and R. M. Swanson, "Stable passivations for high efficiency silicon solar cells,"
IEEE PVSC-90, pp. 318-322, 1990. 